Rev. Int. Contam. Ambie. 35 (3) 723-729, 2019

DOI: 10.20937/RICA.2019.35.03.17

\title{
HORMIGÓN SUSTENTABLE BASADO EN FIBRAS DE NEUMÁTICOS FUERA DE USO
}

Sustainable concrete based on out-of-use tire fibers

\author{
Amin NAZER ${ }^{1,2 *}$, Alexis HONORES ${ }^{1}$, Priscila CHULAK ${ }^{1}$ y Osvaldo PAVEZ ${ }^{2,3}$
}

${ }^{1}$ Departamento de Construcción, Facultad Tecnológica, Universidad de Atacama, Av. Copayapu 485, Copiapó, Chile

${ }^{2}$ Centro Regional de Investigación y Desarrollo Sustentable (CRIDESAT), Universidad de Atacama, Av. Copayapu 485, Copiapó, Chile

${ }^{3}$ Departamento de Ingeniería en Metalurgia, Universidad de Atacama Av. Copayapu 485, Copiapó, Chile

*Autor para correspondencia: amin.nazer@uda.cl

(Recibido noviembre 2017; aceptado septiembre 2018)

Palabras clave: neumáticos usados, hormigón con fibras, valorización de residuos

\section{RESUMEN}

Los neumáticos fuera de uso se consideran residuos masivos y normalmente son abandonados en vertederos legales o ilegales. Su abandono se asocia con impactos medioambientales en el uso del suelo y la contaminación visual, entre otros. Por el contrario, su reciclado permite valorizar estos residuos al generar subproductos tales como gránulos de caucho, fibras de acero y fibras textiles para nuevas aplicaciones industriales. Diversos estudios reportan la utilización de fibras de caucho y de acero de neumáticos fuera de uso en hormigones. El objetivo de esta investigación fue evaluar y comparar la resistencia mecánica de un hormigón sin adición (testigo) a las edades de 7, 17 y 28 días, con hormigones con adición de fibra de acero comercial, fibras de caucho y fibras de acero de neumáticos fuera de uso. Las dosis utilizadas fueron $50 \mathrm{~kg} / \mathrm{m}^{3}$ en hormigones con fibra de acero comercial, 10.5 y $7 \mathrm{~kg} / \mathrm{m}^{3}$ en hormigones con fibras de caucho reciclado, y 50 y $35 \mathrm{~kg} / \mathrm{m}^{3}$ en hormigones con fibras de acero reciclado. Los resultados indicaron que los hormigones con adiciones de fibras de caucho y fibras de acero presentaron en todos los casos una leve disminución de la resistencia a la compresión respecto del testigo a la edad de 28 días. Por el contrario, los hormigones con adiciones de fibras de acero y fibras de caucho presentaron mejor resistencia a la flexo-tracción que el hormigón testigo, excepto el hormigón con dosis de $50 \mathrm{~kg} / \mathrm{m}^{3}$ de fibra de acero reciclado.

Key words: used tires, concrete with fibers, waste recovery

\begin{abstract}
Waste tires are considered to be massive trash and are usually abandoned in legal or illegal landfills. Their abandonment is associated with environmental impacts on land use and visual pollution, among others. Conversely, its recycling allows to valorize these residues by generating by-products such as rubber granules, steel fibers and textile fibers for new industrial applications. Several studies report the use of rubber and steel fibers of waste tires in concrete. The objective of this research was to compare the mechanical
\end{abstract}


strength of concrete without the addition of tires residues (control group) at the ages of 7, 17 and 28 days, to concrete added with commercial steel fiber, and rubber and steel fibers from waste tires. The doses used were $50 \mathrm{~kg} / \mathrm{m}^{3}$ in concrete with commercial steel fiber, 10.5 and $7 \mathrm{~kg} / \mathrm{m}^{3}$ in concrete with recycled rubber granules, and 50 and $35 \mathrm{~kg} / \mathrm{m}^{3}$ in concrete with recycled steel fibers. The results indicated that concretes with the addition of rubber and steel fibers showed in all cases a slight decrease in the compressive strength compared to the witness at the age of 28 days. Conversely, concrete with the addition of steel and rubber fibers presented better flexural strength than the witness concrete, except in the case of concrete with $50 \mathrm{~kg} / \mathrm{m}^{3}$ of recycled steel fiber.

\section{INTRODUCCIÓN}

El creciente uso de medios de transporte a nivel global demanda partes y repuestos, los que al fin de su ciclo de vida son desechados. Los neumáticos fuera de uso (NFU) son piezas descartadas de automóviles, autobuses, camiones, bicicletas y vehículos agrícolas, las cuales se acumulan ocasionalmente en vertederos para su posterior reciclado. Un neumático se fabrica con más de 100 sustancias diferentes, siendo la principal el caucho, con un $50 \%$ del peso total de la pieza, aproximadamente. Además, posee relleno como el negro de humo o gel de sílice $(25 \%$ del peso), acero (10\% del peso), azufre (1\% del peso), óxido de zinc ( $1 \%$ del peso) y muchos otros aditivos como aceite de procesamiento, plastificante o aceleradores de vulcanización, siendo todos estos compuestos difíciles de degradar naturalmente. Los cauchos más comunes utilizados para los neumáticos son el caucho natural, el caucho de estireno-butadieno y el caucho de butadieno (Seidelt et al. 2006).

Anualmente, tanto en la Unión Europea como en Estados Unidos, se depositan alrededor de tres millones de toneladas de NFU (Siddique y Naik 2004, GCR 2017), mientras que en Chile se generan alrededor de $140000 \mathrm{t}$ anuales, de los cuales $50000 \mathrm{t}$ provienen del sector minero. Estos últimos van a lugares de acopio o simplemente se entierran (Morales 2017). En muchos lugares los NFU se consideran residuos sin valor y se depositan en vertederos ilegales afectando negativamente al medio ambiente. Con el propósito de mitigar el impacto medioambiental de los residuos en general, los países cuentan con normativas que regulan su eliminación. En el caso de Chile, en 2016 se dictó la Ley 20.920 (MMA 2016) que establece el marco para la gestión de residuos, la responsabilidad extendida del productor y el fomento al reciclaje; con ello, se responsabiliza a los fabricantes de la organización, recuperación y gestión de sus residuos. Sin embargo, no hay una disposición especial para el manejo de los NFU. En la actualidad, las empresas que reciclan los NFU obtienen subproductos con valor comercial. En la planta recicladora se pueden obtener fibras de caucho, fibras de acero y fibras textiles para diversos usos. Un proceso de reciclado de NFU comprende normalmente las etapas de recepción, selección, trituración, granulación, molienda, almacenamiento y comercialización de los subproductos.

Diversos estudios reportan la utilización de fibras de acero y de caucho de NFU en hormigones para uso estructural y no estructural. Aiello et al. (2009) estudiaron hormigones con adición de fibras de acero (FA) de NFU en dosis bajas, obteniendo un incremento en la resistencia a la flexión respecto del hormigón testigo. Además, Graeff et al. (2012) concluyeron que la adición de 2 y $6 \%$ en peso de FA en el hormigón mejoró el comportamiento resistente de pavimentos, llegando incluso a reducir su espesor hasta en un $26 \%$. Por otra parte, Siddique y Naik (2004) reportaron que los hormigones con fibras de caucho (FC) de NFU mejoran la adherencia con la pasta de cemento adicionando oxicloruro de magnesio. Además, Mohammadi y Khabbaz (2015) y Serdar et al. (2015) concluyeron que la adición de $\mathrm{FC}$ en pavimentos reduce las grietas de retracción.

El objetivo de esta investigación fue evaluar comparativamente la resistencia mecánica de hormigones con adición de fibra de acero comercial y hormigones con FC y FA, ambos de NFU, con un hormigón sin adición (testigo) a las edades de 7, 17 y 28 días.

\section{MATERIALES Y MÉTODOS}

\section{Materiales}

En la fabricación de las muestras se utilizó hormigón premezclado del proveedor Ready Mix, el cual fue elaborado con cemento Portland puzolánico de alta resistencia inicial, de acuerdo con la Norma Chilena 148 Of.1968 (INN 1968), tamaño máximo del árido grueso de $20 \mathrm{~mm}$ y asentamiento de cono de 
$16 \mathrm{~cm}$, para una resistencia mínima a la compresión de 30 Mpa a los 28 días. Además, el hormigón premezclado contiene aditivo plastificante Plastocrete ${ }^{\circledR}$ CB $400 \mathrm{R}$, del fabricante SIKA y aditivo inhibidor de hidratación Delvo ${ }^{\circledR}$ del fabricante BASF. En el cuadro I se muestra la distribución de tamaño de partículas de los áridos utilizados en la fabricación del hormigón, y en el cuadro II algunas de las propiedades físicas y químicas de estos áridos. Además, en el cuadro III se especifican las propiedades del cemento entregadas por el proveedor. Para la fabricación de los hormigones se utilizó agua potable de la ciudad de Tierra Amarilla; FA y FC obtenidos por procesos mecánicos por la empresa recicladora chilena de NFU Polambiente S.A., y fibra de acero comercial Dramix ${ }^{\circledR}$ 3D del fabricante Bekaert, esta última con longitudes de $60 \mathrm{~mm}$ y diámetro 0.75 mm (Fig. 1). En la figura 2 se muestran las FA de NFU de 35 a $50 \mathrm{~mm}$ de longitud con diámetros de $0.89 \mathrm{~mm}$ aproximadamente. Además, en la figura 3 se muestran las FC de NFU con tamaños de partícula de 0.1 a $5 \mathrm{~mm}$ utilizados en la fabricación de los hormigones, y en la figura 4 se puede observar la distribución granulométrica de FC de NFU, en la que predomina el tamaño $2.5 \mathrm{~mm}$ con un $88 \%$.

CUADRO I. DISTRIBUCIÓN DE TAMAÑO DE PARTÍCULAS DE LOS ÁRIDOS

\begin{tabular}{lcc}
\hline Tamaño $(\mathrm{mm})$ & Gravilla (\% pasa) & Arena (\% pasa) \\
\hline 25 & & \\
20 & 100 & \\
12.5 & 64 & \\
10 & 43 & 100 \\
5 & 9 & 83 \\
2.5 & & 63 \\
1.25 & & 54 \\
0.63 & & 35 \\
0.315 & & 18 \\
0.16 & & 4 \\
\hline
\end{tabular}

\section{CUADRO II. PROPIEDADES DE LOS ÁRIDOS}

\begin{tabular}{lcc}
\hline Propiedad & Gravilla & Arena \\
\hline Densidad aparente suelta $\left(\mathrm{kg} / \mathrm{m}^{3}\right)$ & 1413 & 1746 \\
Densidad real $\left(\mathrm{kg} / \mathrm{m}^{3}\right)$ & 2674 & 2618 \\
Humedad $(\%)$ & 0.4 & 2.7 \\
Absorción (\%) & 0.9 & 0.9 \\
Fino tamaño menor que $0.8 \mathrm{~mm}(\%)$ & 0.2 & 3.0 \\
$\mathrm{SO}_{4}{ }^{=}(\mathrm{kg} / \mathrm{kg}$ de árido) & $3.14 \times 10^{-4}$ & $3.29 \mathrm{E}-5$ \\
$\mathrm{Cl}^{=}(\mathrm{kg} / \mathrm{kg}$ de árido) & $3.32 \times 10^{-5}$ & $1.08 \mathrm{E}-04$ \\
\hline
\end{tabular}

CUADRO III. PROPIEDADES DEL CEMENTO PORTLAND

\begin{tabular}{lc}
\hline Peso específico $\left(\mathrm{kg} / \mathrm{m}^{3}\right)$ & 3030 \\
Superficie específica $\left(\mathrm{cm}^{2} / \mathrm{g}\right)$ & 4150 \\
Inicio de fraguado $(\mathrm{h})$ & 3 \\
Término de fraguado $(\mathrm{h})$ & 4 \\
Pérdida por calcinación $(\%)$ & 1.4 \\
Contenido de $\mathrm{SO}_{3}(\%)$ & 2.56 \\
Residuo insoluble $(\%)$ & 14.2 \\
\hline
\end{tabular}

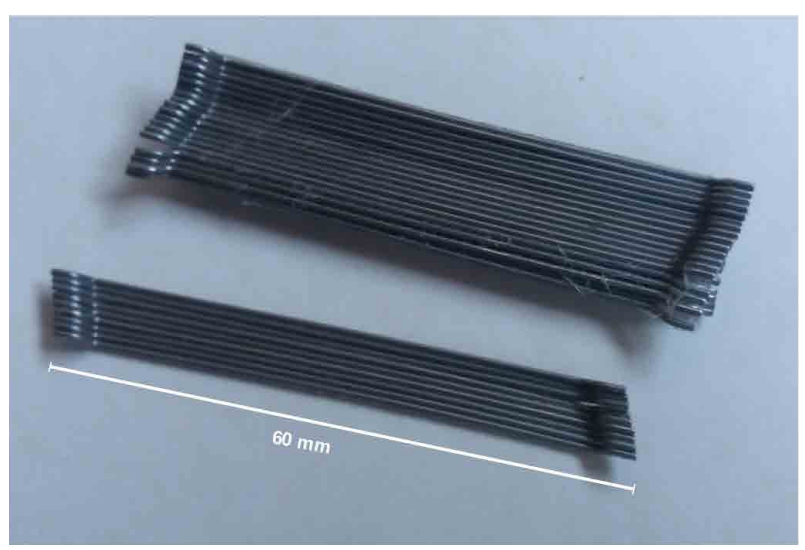

Fig. 1. Fibra de acero comercial utilizada en hormigones

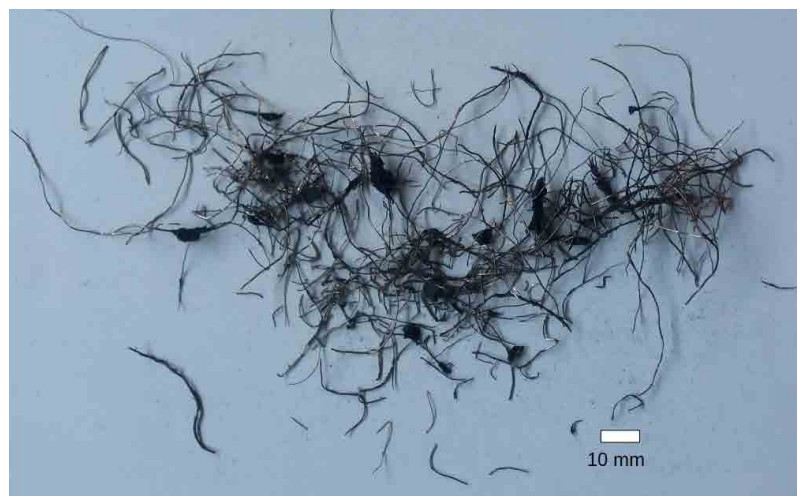

Fig. 2. Fibras de acero de neumáticos fuera de uso utilizadas en hormigones

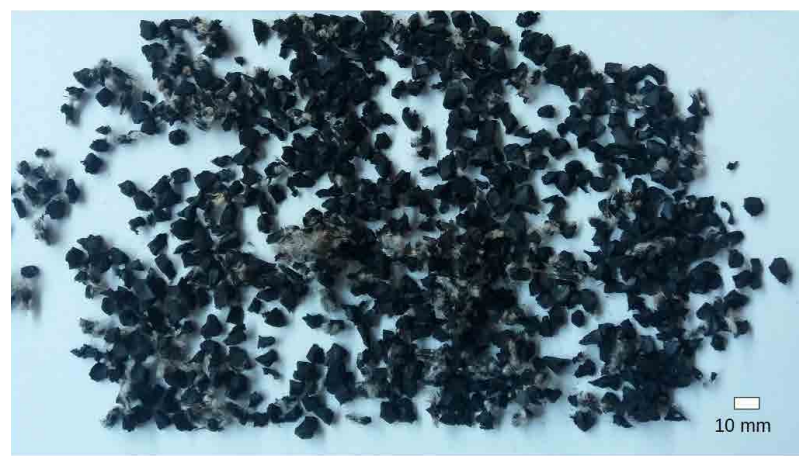

Fig. 3. Fibras de caucho de neumáticos fuera de uso utilizadas en hormigones 


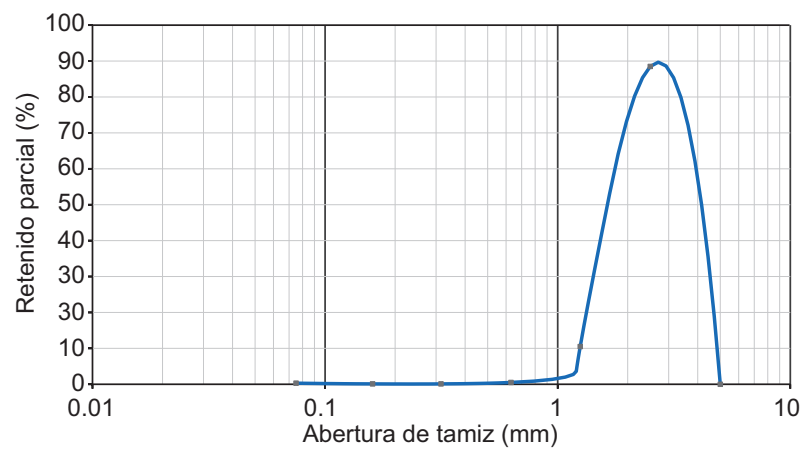

Fig. 4. Distribución de tamaño de partículas de fibras de caucho de neumáticos fuera de uso

\section{Métodos}

Se fabricaron por triplicado seis diferentes tipos de hormigón, evaluando su resistencia a la compresión a las edades de 7, 17 y 28 días y a la flexotracción a los 28 días. Las adiciones utilizadas fueron de $50 \mathrm{~kg} / \mathrm{m}^{3}$ en hormigones con fibra de acero comercial DRAMIX $^{\circledR}$ (FD50), y de 35 (FA35) y $50 \mathrm{~kg} / \mathrm{m}^{3}$ (FA50) en hormigones con FA de NFU. Además, en hormigones con FC de NFU se utilizaron adiciones en dosis de $7 \mathrm{~kg} / \mathrm{m}^{3}$ (FC7) y $10.5 \mathrm{~kg} / \mathrm{m}^{3}$ (FC10.5). Los hormigones con fibras fueron comparados con una muestra sin adiciones que sirvió de testigo. Las fibras FA y FC se incorporaron gradualmente al hormigón premezclado antes de ser vertidas a los moldes. Para ello, se procedió al remezclado del hormigón fabricado en planta, con FA y FC en mezcladora eléctrica durante $1.5 \mathrm{~min}$, asegurando la distribución homogénea de los componentes de acuerdo con la norma NCh 1789 Of.1986 (INN 1986). Posteriormente las muestras moldeadas fueron compactadas mediante vibración con sonda, desmoldadas a las $24 \mathrm{~h}$ y sumergidas en agua con cal hasta la edad de ensayo. Se utilizaron probetas de $20 \times 20 \times 20 \mathrm{~cm}^{3}$ para ensayos a la compresión y probetas de $15 \times 15 \times 53 \mathrm{~cm}^{3}$, con luz de ensayo de $450 \mathrm{~mm}$, en flexotracción. La rotura de las muestras se realizó en prensas del fabricante Controls. En el cuadro IV se identifican los tipos de hormigones con las adiciones de fibras recicladas de NFU estudiados y en el cuadro $\mathbf{V}$ la dosificación utilizada para la fabricación del hormigón sin adición.

\section{RESULTADOS Y DISCUSIÓN}

El cuadro VI y la figura 5 muestran la resistencia mecánica del hormigón testigo y de los hormigones con FA y FC. Los resultados de la resistencia a compresión a la edad de 28 días de los hormigones
CUADRO IV. IDENTIFICACIÓN DE LAS MUESTRAS Y DOSIS DE FIBRAS ADICIONADAS

\begin{tabular}{lccc}
\hline ID Muestra & $\begin{array}{c}\text { FA } \\
\left(\mathrm{kg} / \mathrm{m}^{3}\right)\end{array}$ & $\begin{array}{c}\mathrm{FC} \\
\left(\mathrm{kg} / \mathrm{m}^{3}\right)\end{array}$ & $\begin{array}{c}\mathrm{FD} \\
\left(\mathrm{kg} / \mathrm{m}^{3}\right)\end{array}$ \\
\hline Testigo & - & - & - \\
FA35 & 35 & - & - \\
FA50 & 50 & - & - \\
FD50 & - & - & 50 \\
FC7 & - & 7 & - \\
FC10.5 & - & 10.5 & - \\
\hline
\end{tabular}

FA: fibras de acero, FC: fibras de caucho, FD: fibra de acero comercial DRAMIX $^{\circledR}$

CUADRO V. DOSIS DE MATERIALES UTILIZADOS PARA $1 \mathrm{~m}^{3}$ DE HORMIGÓN

\begin{tabular}{llc} 
Material & Característica & Cantidad \\
\hline Cemento (kg) & $\begin{array}{l}\text { Portland puzolánico, } \\
\text { grado alta resistencia }\end{array}$ & 296 \\
\hline Arena (kg) & De río & 1010 \\
\hline Gravilla (kg) & Triturada & 818 \\
\hline Agua (L) & Potable & 206 \\
\hline $\begin{array}{l}\text { Aditivo plasti- } \\
\text { ficante (kg) }\end{array}$ & Plastocrete CB 400 R, $^{\circledR}$ SIKA $^{\circledR}$ & 1.48 \\
\hline $\begin{array}{l}\text { Aditivo inhibidor } \\
\text { hidratación (L) }\end{array}$ & Delvo $^{\circledR}$ & 2.23 \\
\hline
\end{tabular}

con fibras de NFU reciclado, están comprendidos entre 31 y $34 \mathrm{Mpa}$, aproximadamente. En general, como puede observarse en la figura $\mathbf{6}$, los resultados a la compresión son levemente inferiores respecto del hormigón testigo ( $35 \mathrm{Mpa}$ ), con reducciones de 3 a $11 \%$. Además, los hormigones con fibras evidencian un comportamiento a la flexotracción similar, y en algún caso superior al del testigo. El análisis de los datos indica que la resistencia a la flexotracción de los hormigones con fibras (a excepción de FA50 con $11 \%$ ) son 3 a $26 \%$ superiores al testigo, destacando la muestra FD50 con $4.8 \mathrm{Mpa}$. En la figura 5 también se puede observar que a medida que aumenta la adición de FA de NFU, disminuye levemente su resistencia tanto a compresión como a flexotracción a los 28 días, comportamiento que puede estar asociado a la disminución de la docilidad de la mezcla, hecho que dificulta la maleabilidad y la compactación adecuada de la misma. Por otra parte, los hormigones con adición de FC, presentan un leve incremento en la resistencia a la 
CUADRO VI. COMPORTAMIENTO RESISTENTE DE HORMIGONES CON FIBRAS

\begin{tabular}{|c|c|c|c|c|c|c|c|c|}
\hline \multirow{3}{*}{ Muestra } & \multicolumn{3}{|c|}{ Densidad aparente $\left(\mathrm{kg} / \mathrm{m}^{3}\right)$} & \multirow{3}{*}{$\begin{array}{l}\text { Docilidad } \\
\quad(\mathrm{cm})\end{array}$} & \multirow{2}{*}{\multicolumn{3}{|c|}{$\begin{array}{c}\text { Compresión (MPa) } \\
\text { Edad (días) }\end{array}$}} & \multirow{3}{*}{$\begin{array}{c}\text { Flexotracción (MPa) } \\
\text { Edad (días) } \\
28\end{array}$} \\
\hline & \multicolumn{3}{|c|}{ Edad (días) } & & & & & \\
\hline & 7 & 17 & 28 & & 7 & 17 & 28 & \\
\hline Testigo & 2360 & 2330 & 2300 & 18.0 & 27.1 & 29.9 & 35.3 & 3.8 \\
\hline FA35 & 2360 & 2360 & 2350 & 8.0 & 26.1 & 29.9 & 34.3 & 3.9 \\
\hline FA50 & 2370 & 2400 & 2380 & 5.0 & 26.0 & 30.9 & 33.4 & 3.4 \\
\hline FD50 & 2340 & 2350 & 2340 & 7.0 & 24.0 & 28.1 & 30.6 & 4.8 \\
\hline FC7 & 2330 & 2330 & 2330 & 18.5 & 26.7 & 28.1 & 30.7 & 3.9 \\
\hline FC10.5 & 2330 & 2310 & 2330 & 18.0 & 23.6 & 28.6 & 33.9 & 4.4 \\
\hline
\end{tabular}

FA35: $35 \mathrm{~kg} / \mathrm{m}^{3}$ de adición de fibras de acero, FA50: $50 \mathrm{~kg} / \mathrm{m}^{3}$ de adición de fibras de acero, FD50: $50 \mathrm{~kg} / \mathrm{m}^{3}$ de adición de fibras de acero comercial DRAMIX ${ }^{\circledR}, F C 7: 7 \mathrm{~kg} / \mathrm{m}^{3}$ de adición de fibras de acero, FC10.5: $10.5 \mathrm{~kg} / \mathrm{m}^{3}$ de adición de fibras de caucho

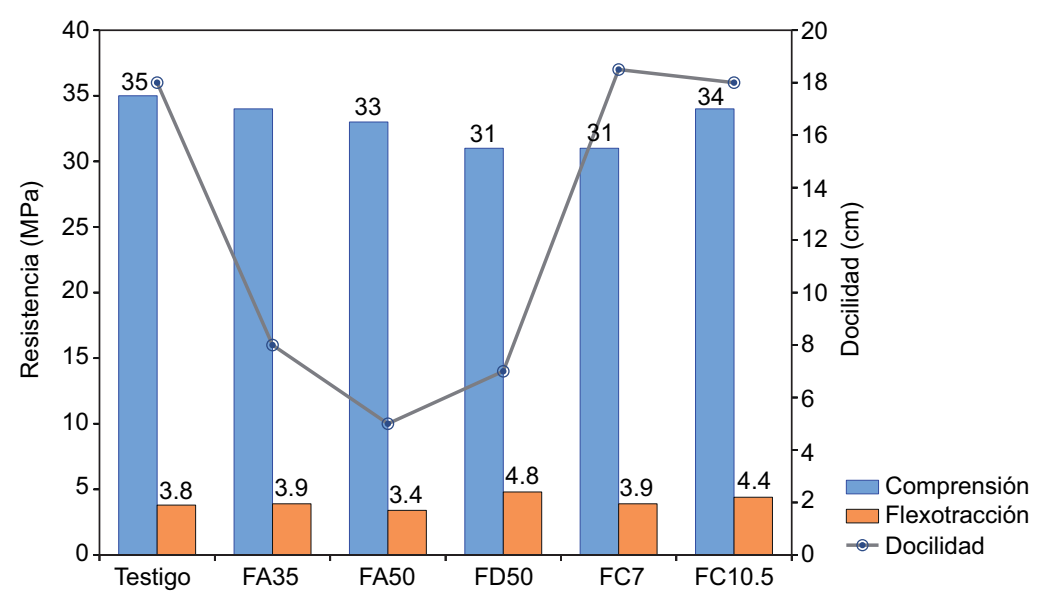

Fig. 5. Resistencias a la compresión y flexotracción a los 28 días y docilidad de las muestras. FA: fibras de acero, FD: fibras de acero comercial DRAMIX ${ }^{\circledR}$, FC: fibras de caucho

compresión a los 28 días, mientras que la resistencia a la flexotracción de la muestra FC10.5 es superior a FC7. Como se puede observar en el cuadro VI, la resistencia a la compresión de las muestras para todos los casos aumenta con la edad de curado, alcanzando su máximo a los 28 días.

En cuanto a la resistencia esperada a mayor edad de curado, situación no contemplada en el presente estudio, autores como Aiello et al. (2009) ensayaron muestras de hormigón con adición de fibras de acero de NFU y compararon la resistencia a la compresión a la edad de 60 días con una muestra testigo. Los resultados indicaron que para diferentes docilidades del hormigón testigo, y para hormigones que contenían fibras de NFU, la resistencia a la compresión fue de $32 \mathrm{MPa}$, similar a lo obtenido en el presente estudio, mientras que en los hormigones con fibras de acero reciclado que contenían 0.23 y $0.46 \%$ en volumen, y en hormigones con $0.40 \%$ en volumen de fibras comerciales, se alcanzó una resistencia de $38 \mathrm{MPa}$. Los resultados obtenidos tanto por los autores del presente estudio como otros autores, muestran un incremento de la resistencia de los hormigones que contienen fibras de NFU a medida que aumenta la edad de curado. Esto se confirma con los estudios de Graeff et al. (2009), quienes concluyeron que las muestras que contenían FA de NFU ensayadas a edades mayores a 4 meses, mejoraron sus propiedades resistentes incluso por sobre la resistencia del hormigón testigo. El comportamiento resistente de los hormigones con adición de fibras de NFU estudiados permite sugerir su uso en obras viales, principalmente 
en pavimentos (Graeff et al. 2012, Mohammadi y Khabbaz 2015, Serdar et al. 2015). Sin embargo, para su utilización en pavimentos, se requiere mayor estudio tanto en laboratorio (ensayos de durabilidad, fatiga, compactación con rodillo, entre otros), como en terreno, mediante canchas de prueba in situ y con tráfico normal de vehículos.

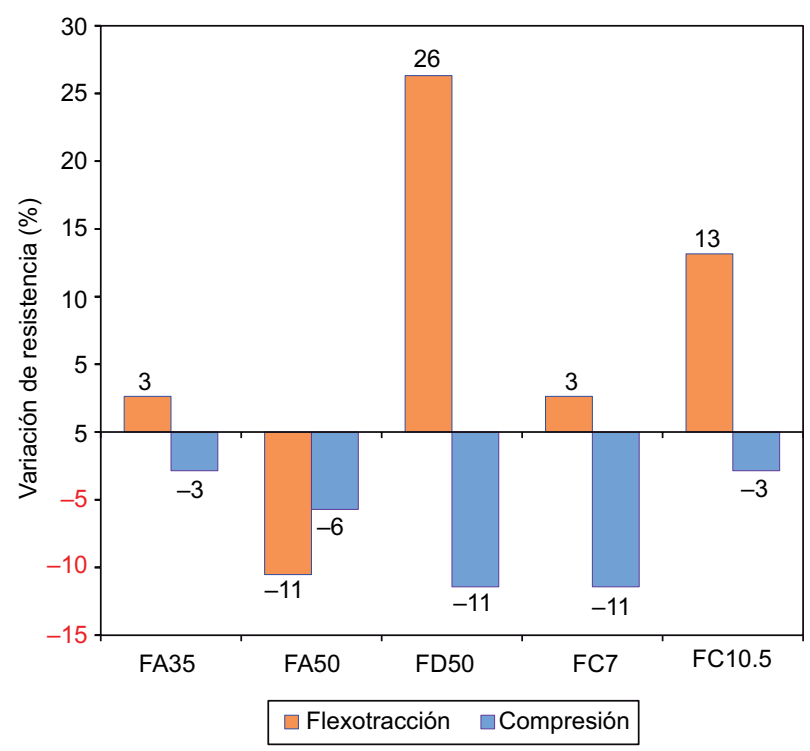

Fig. 6. Variación de la resistencia a compresión y flexotracción, respecto del testigo a los 28 días. FA: fibras de acero, FD: fibras de acero comercial DRAMIX ${ }^{\circledR}$, FC: fibras de caucho

\section{CONCLUSIONES}

En este estudio, se verificó el comportamiento mecánico de muestras de hormigón que contenían FA y FC de NFU. En los ensayos de compresión y flexotracción, se constató que el hormigón testigo presentó el mejor comportamiento a la compresión a los 28 días; sin embargo, las muestras FA35 y FC10.5 estuvieron ligeramente por debajo del testigo, lo que pone de manifiesto que es viable fabricar hormigones resistentes a la compresión con adiciones de FA y FC de NFU en proporciones de hasta 35 y $10.5 \mathrm{~kg} / \mathrm{m}^{3}$, respectivamente. Por otra parte, el comportamiento resistente de las muestras con fibras ensayadas a flexotracción, mostraron en general mejores resultados que el hormigón testigo, lo que sugiere la utilización de FA y FC de NFU en cantidades inferiores a 50 y $10.5 \mathrm{~kg} / \mathrm{m}^{3}$, respectivamente. Como conclusión general, el estudio indicó que la adición de fibras de NFU en mezclas basadas en cemento sugiere la posibilidad de su utilización en la fabricación de hormigones, particularmente en pavimentos, valorizando un residuo habitual que de otra forma podría terminar en el vertedero por un tiempo indefinido.

\section{REFERENCIAS}

Aiello M., Leuzzi F., Centonze G. y Maffezzoli A. (2009). Use of steel fibres recovered from waste tyres as reinforcement in concrete: Pull-out behaviour, compressive and flexural strength. Waste Manage. 29 (6), 19601970. DOI: $10.1016 /$ j.wasman.2008.12.002

GCR (2017). Old tyres can make bendy buildings that could better withstand earthquakes. Global Construction Review [en línea]. http:/www.globalconstructionreview.com/news/old-tyres-can-m8ake-bend6yb2ui0ldi8n6gs-4c2o0u8ld/ 02/05/2018

Graeff A., Pilakoutas K., Neocleous K. y Peres M.V.N. (2012). Fatigue resistance and cracking mechanism of concrete pavements reinforced with recycled steel fibres recovered from post-consumer tyres. Eng. Struct. $45,385-395$.

DOI: 10.1016/J.ENGSTRUCT.2012.06.030

INN (1968). Norma Chilena Oficial NCh148.Of1968. Cemento - Terminología, clasificación y especificaciones generales. Instituto Nacional de Normalización. Decreto $N^{\circ} 446$ del Ministerio de Obras Públicas y Transportes, Santiago de Chile, Chile, 7 de mayo.

INN (1986). Norma Chilena Oficial NCh1789.Of1986. Hormigón - Determinación de la uniformidad obtenida en el mezclado del hormigón fresco. Instituto Nacional de Normalización. Decreto $\mathrm{N}^{\mathrm{o}} 17$ del Ministerio de Vivienda y Urbanismo, Santiago de Chile, Chile, 30 de enero.

MMA (2016) Ley $\mathrm{N}^{\circ}$ 20.920. Establece marco para la Gestión de Residuos, la Responsabilidad extendida al productor y Fomento al Reciclaje. Ministerio del Medio Ambiente, Santiago de Chile, Chile, 17 de mayo.

Morales C. (2017). Por un nuevo modelo en reciclaje de materiales. Nueva Minería y Energía 98, 58-60.

Mohammadi I. y Khabbaz H. (2015). Shrinkage performance of crumb rubber concrete (CRC) prepared by water-soaking treatment method for rigid pavements. Cement Concrete Comp. 62, 106-116. DOI: 10.1016/J.CEMCONCOMP.2015.02.010

Seidelt S., Müller-Hagedorn M. y Bockhorn H. (2006). Description of tire pyrolysis by thermal degradation behaviour of main components. J. Anal. Appl. Pyrol. 75 (1), 11-18.

DOI: 10.1016/J.JAAP.2005.03.002 
Serdar M., Baričević A., Jelčić Rukavina M., Pezer M., Bjegović D. y Štirmer N. (2015). Shrinkage behaviour of fibre reinforced concrete with recycled tyre polymer fibres. Int. J. Polym. Sci. 2015, ID 145918.

DOI: $10.1155 / 2015 / 145918$
Siddique R. y Naik T.R. (2004). Properties of concrete containing scrap-tire rubber - an overview. Waste Manage. 24 (6), 563-569.

DOI: 10.1016/J.WASMAN.2004.01.006 